\title{
Production of the Bengamide Class of Marine Natural Products in Myxobacteria: Biosynthesis and Structure-Activity Relationships
}

\author{
Silke C. Wenzel, Holger Hoffmann, Jidong Zhang, Laurent Debussche, Sabine Haag-Richter, \\ Michael Kurz, Frederico Nardi, Peer Lukat, Irene Kochems, Heiko Tietgen, Dietmar Schummer, \\ Jean-Paul Nicolas, Loreley Calvet, Valerie Czepczor, Patricia Vrignaud, Agnes Mühlenweg, \\ Stefan Pelzer, Rolf Müller,* and Mark Brönstrup*
}

\begin{abstract}
The bengamides, sponge-derived natural products that have been characterized as inhibitors of methionine aminopeptidases (MetAPs), have been intensively investigated as anticancer compounds. We embarked on a multidisciplinary project to supply bengamides by fermentation of the terrestrial myxobacterium M. virescens, decipher their biosynthesis, and optimize their properties as drug leads. The characterization of the biosynthetic pathway revealed that bacterial resistance to bengamides is conferred by Leu154 of the myxobacterial MetAP protein, and enabled transfer of the entire gene cluster into the more suitable production host M. xanthus DK1622. A combination of semisynthesis of microbially derived bengamides and total synthesis resulted in an optimized derivative that combined high cellular potency in the nanomolar range with high metabolic stability, which translated to an improved half-life in mice and antitumor efficacy in a melanoma mouse model.
\end{abstract}

$\mathbf{N}$ animals traditionally play an important role in biomedical research, as the majority of antibacterial and cytotoxic anticancer drugs in clinical use are either such secondary metabolites or derivatives thereof. ${ }^{[1]}$ Although the marine environment represents an enormous additional reservoir for novel secondary metabolites, ${ }^{[2]}$ the development of marine natural products as drugs is hampered by difficulties in establishing a sustainable and scalable compound supply. The supply problem can be either overcome through chemical synthesis, as exemplified by eribulin ${ }^{[3]}$ and trabectedin, ${ }^{[4]}$ or through the identification of a cultivable microorganism as a production source, as based on a growing body of evidence that the "true" producer of many natural products discovered from marine sources is a symbiotic microorganism. ${ }^{[5]}$

A prominent class of marine natural products is formed by the bengamides, first isolated in 1986 from the marine sponge Jaspis cf. coraciae. ${ }^{[6]}$ As a consequence of their nanomolar potency in the NCI-60 human tumor cell lines screen, bengamide B (1) and other analogues were intensively investigated as anticancer lead compounds (Figure 1). An efficient synthetic route ${ }^{[7]}$ enabled the optimization of the lead compound $\mathbf{1}$ to yield LAF389 (2), ${ }^{[8]}$ which was studied in an anticancer phase I clinical trial, but not further developed as a result of cardiovascular side effects. ${ }^{[9]}$ As the molecular targets of bengamides, the human methionine aminopeptidases (MetAPs) ${ }^{[10]}$ are attractive anticancer targets, ${ }^{[11]}$ and an additional use as compounds to treat tuberculosis has been proposed ${ }^{[12]}$ the search for improved analogues is intensively pursued. ${ }^{[6 c, 13]}$

Herein, we report the outcome of a multidisciplinary project on bengamides that was triggered by our serendipitous discovery of the terrestrial myxobacterium Myxococcus virescens ST200611 (DSM 15898) as a producer of bengamides. ${ }^{[14]}$ The gram-scale fermentative access to this class of "marine" natural products allowed studies to be conducted on the biosynthesis of bengamides, their heterologous expression, and the self-resistance mechanism of their producer. We also optimized their properties as drug leads by semi- and total synthesis. ${ }^{[15]}$ Johnston et al. recently also reported the

[*] Dr. S. C. Wenzel, Dr. P. Lukat, I. Kochems, Prof. Dr. R. Müller Helmholtz Institut für Pharmazeutische Forschung Saarland, Helmholtz Zentrum für Infektionsforschung and Universität des Saarlandes 66123 Saarbrücken (Germany) E-mail: Rolf.Mueller@helmholtz-hzi.de

Dr. H. Hoffmann, Dr. J. Zhang, Dr. L. Debussche, Dr. S. Haag-Richter, Dr. M. Kurz, Dr. F. Nardi, Dr. H. Tietgen, J.-P. Nicolas, Dr. L. Calvet, Dr. V. Czepczor, Dr. P. Vrignaud Sanofi R\&D, Industriepark Hoechst 65926 Frankfurt (Germany) and 13 Quai Jules Guesde, Vitry sur Seine 94403 (France) Prof. Dr. D. Schummer TH Mittelhessen Wiesenstrasse 14, 35390 Gießen (Germany)

\author{
Priv.-Doz. Dr. S. Pelzer \\ Evonik Nutrition \& Care \\ Kantstrasse 2, 33790 Halle (Germany) \\ Dr. A. Mühlenweg \\ Technische Universität Berlin \\ Str.d.17. Juni 124, 10623 Berlin (Germany) \\ Prof. Dr. M. Brönstrup \\ Helmholtz Zentrum für Infektionsforschung \\ Inhoffenstrasse 7, 38124 Braunschweig (Germany) \\ E-mail: Mark.Broenstrup@helmholtz-hzi.de \\ (D) Supporting information for this article (including data on the \\ microbial production, isolation, structural characterization, biologi- \\ cal testing, gene cluster characterization and engineering, and on \\ synthetic procedures) is available on the WWW under http://dx.doi. \\ org/10.1002/anie.201508277.
}




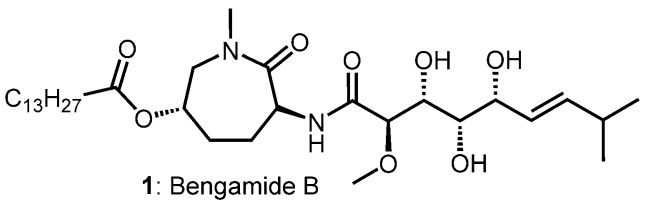<smiles>COC(C(=O)NC1CCC(OC(=O)C2CCCCC2)CN(C)C1=O)[C@H](O)[C@@H](O)[C@H](O)/C=C/C(C)(C)C</smiles><smiles>[R][Z]C(C)C=C[C@@H](O)[C@@H](O)[C@@H](O)[C@H](OC)C(=O)N[C@H]1CCCCN([R1])C1=O</smiles>

3: $R^{1}=H, R^{2}=M e$, Bengamide $E^{\prime}$

4: $R^{1}=M e, R^{2}=M e$, Bengamide $F^{\prime}$

5: $R^{1}=H, R^{2}=H$, Bengamide $E$

6: $R^{1}=\mathrm{Me}, \mathrm{R}^{2}=\mathrm{H}$, Bengamide $\mathrm{F}$<smiles>CC[C@H](C)/C=C/[C@H](O)[C@@H](O)[C@H](O)[C@H](OC)C(=O)NC1CCCCN(Cc2ccccc2)C1=O</smiles>

$7 a$

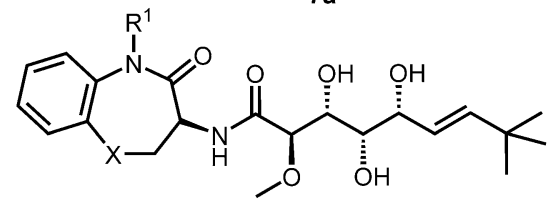

8a: $\mathrm{R}^{1}=\mathrm{H}, \mathrm{X}=\mathrm{CH}_{2}$

8d: $R^{1}=M e, X=0$

Figure 1. Chemical structure of bengamides. 1 and 3-6 have been isolated from natural sources, while $\mathbf{2}, \mathbf{7 a}, \mathbf{8 a}$, and $8 \mathrm{~d}$ have been obtained by synthesis. The structures of $7 \mathbf{b}-\mathbf{e}$, derivatives with substituted $N$-benzyl residues, can be found in the Supporting Information.

production of bengamides by the same strain of $M$. virescens and characterized the potent anti-inflammatory properties of the compound class. ${ }^{[16]}$

To exploit myxobacteria as a source of original bioactive chemical matter, ${ }^{[17]} M$. virescens ST200611 was isolated from a soil sample and cultivated (see Figure S1 in the Supporting Information). Compounds 3-6 were isolated on a preparative scale in $>95 \%$ purity in amounts of $145 \mathrm{mg}, 35 \mathrm{mg}, 86 \mathrm{mg}$, and $<1 \mathrm{mg}$, respectively, from the extract of a $30 \mathrm{~L}$ fermentation. ${ }^{[14]}$ Multidimensional NMR and mass spectrometry experiments revealed that 3-6 belong to the bengamide class of (hitherto marine) natural products (Figure 1, see also Figures S2 and S3, and Table S1). Although $\mathbf{5}$ and $\mathbf{6}$ were also isolated from marine sources, $\mathbf{3}$ or $\mathbf{4}$ carried an additional methyl group at $\mathrm{C} 17$. An analysis of the antiproliferative activities of $\mathbf{3}-\mathbf{5}$ in 8 cell lines showed that most $\mathrm{IC}_{50}$ values were in the low micromolar range (Table S2), 2-3 orders of magnitudes higher than that of the marine product $\mathbf{1}$ or the optimized analogue 2 ; this clearly implied that improvements through structural alterations were necessary.

We next aimed to decipher the genetic blueprint for bengamide biosynthesis to exploit this information to improve the structure and yield. A polyketide synthase $(\mathrm{PKS})^{[18]} /$ nonribosomal peptide synthetase (NRPS) ${ }^{[19]}$ hybrid system was identified and shown to be involved in bengamide production by targeted mutagenesis (see the Supporting Information). Gene cluster borders were deduced by comparison with Myxococcus xanthus genome data, which indicated that the biosynthetic pathway spans about $25 \mathrm{kbp}$ and consists of nine genes, ben $A-I$ (Figure 2, see also Figure S4 and Table S3).

Bengamide assembly is catalyzed by a modular megasynthetase (BenA-D), which is supplied with glycerolderived hydroxymalonyl carrier protein (CP) extender units $^{[20]}$ by BenE-H. Results of a feeding experiment with $\left[2-{ }^{13} \mathrm{C}\right]$ glycerol corroborate the incorporation of two such units by modules 2 and 3 (Figure S5). Based on this finding, the following biosynthesis is proposed: Isobutyryl-CoA or 2-methylbutyryl-CoA starter units are extended with malonyl-CoA, two "glycolate units", and finally L-lysine. Additional reductive polyketide chemistry, $O$-methylation, and the ultimate release of the PK/NRP chain from the enzyme complex by lactamization results in the formation of $\mathbf{3}$ and $\mathbf{5}$. The generated caprolactam moiety is optionally further modified by $N$-methylation to yield 4 and $\mathbf{6}$. As no putative $\mathrm{N}$-methyl transferase gene was identified in the cluster region, the required enzyme activity is assumed to be encoded elsewhere in the genome.

In addition to the Ben $\mathrm{A}-\mathrm{H}$ biosynthesis machinery, a putative methionine aminopeptidase (BenI) is encoded within the bengamide gene cluster (Table S3). Myxobacterial genomes usually encode two copies of MetAPs (MetAP1a and MetAP1b; Figure S9). BenI in M. virescens ST20011 represents an additional replica of MetAP1b; this indicates a possible resistance-conferring function, since bengamides are MetAP inhibitors. ${ }^{[10]}$ Sequence analysis revealed a leucine residue in position 154, where other prokaryotic and eukaryotic MetAPs usually harbor a conserved cysteine or alanine residue (Figure S9). A homology model indicated a steric clash between the more bulky leucine residue and the bengamide methoxy group, thus rationalizing resistance (Figure 3). To verify this hypothesis, growth inhibition studies with bengamide-sensitive Escherichia coli strains expressing BenI and a Leu154Cys-mutated version were performed (Figure S9). The obtained data strongly suggest that BenI mediates resistance against bengamides, and that Leu 154 plays a major role in preventing inhibition.

To further exploit the identified biosynthetic pathway, we next aimed to establish a heterologous production system, as genetic tools for the native bengamide producer were limited. After initial attempts to express the pathway in streptomycetes failed, the genetic construct was modified for transfer into the myxobacterial model strain M.xanthus DK1622 (pBen32; Figure S6). Expression under control of the native promoters resulted in the production of all four bengamide derivatives known from $M$. virescens ST200611 in a total yield 

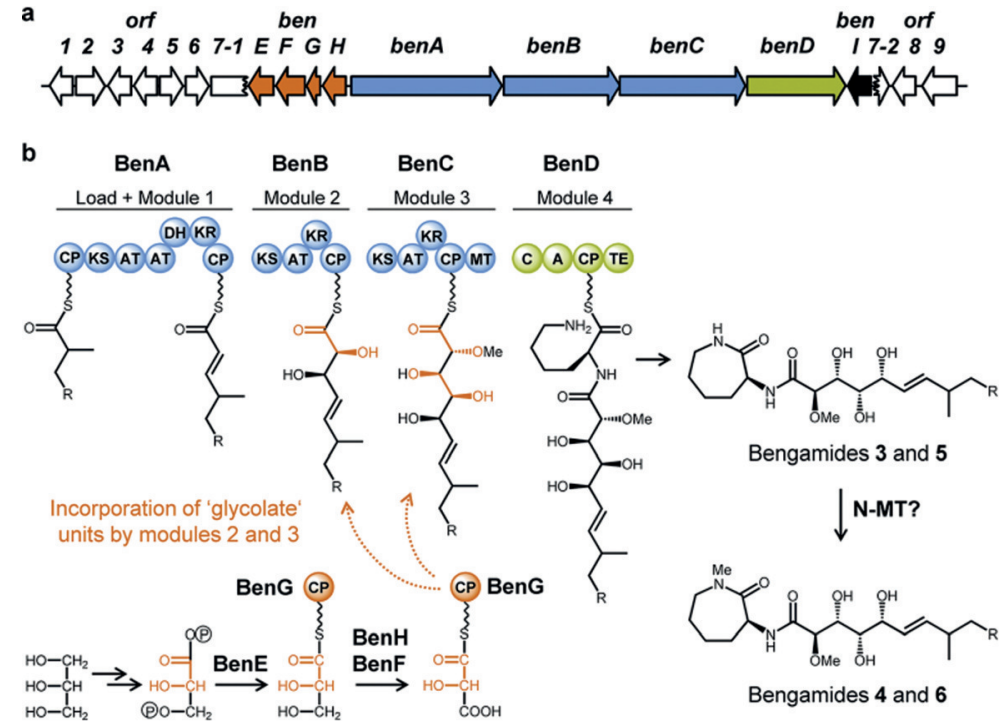

Figure 2. Bengamide biosynthesis in M. virescens ST200611. A) Bengamide biosynthetic gene cluster (benA-l) with flanking regions. B) Model for bengamide assembly by a hybrid polyketide/nonribosomal peptide megasynthetase (PKS parts blue, NRPS parts green). See also Table S3.
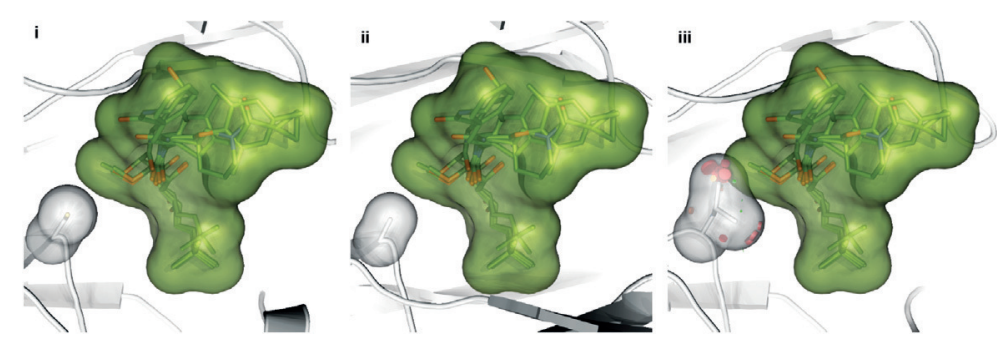

Figure 3. In silico analysis of Benl compared to crystal structures of other methionine aminopeptidases (MetAPs) with the superposition of five bengamide inhibitors (green) from MetAP co-structures available in the PDB. i) Mycobacterium tuberculosis MetAP (PDB: 3PKA). ${ }^{[12]}$ ii) Human MetAP (PDB: 1QZY). ${ }^{[10]}$ iii) Homology model of Benl. The most probable rotamer for L154 is shown in a stick and surface representation and is in the equivalent position to A230 in human MetAP and to $\mathrm{C} 113$ in the M. tuberculosis protein. Steric clashes between the L154 side chain and the methoxy group of the bengamide inhibitors are symbolized by disks and seem to occur for all possible rotamers. For details see Figure S10.

of $5-10 \mathrm{mg} \mathrm{L}^{-1}$ (Figure S8). The achieved production titer was comparable to the native producer and represents a very promising result for such initial heterologous expression studies, thus setting the stage for flexible modification of the pathway. Although a first genetic engineering approach (deletion of the benE-H operon; see the Supporting Information) has not yet resulted in the production of novel bengamide derivatives, the established heterologous expression system represents a highly valuable platform for future improvement of the structure and yield. The system will, for example, enable modifications within the $731 \mathrm{kDa}$ bengamide megasynthetase that are not feasible in the native producer.

To enhance the antiproliferative potency of bengamides, 3 was produced in gram quantities by fermentation and modified by semisynthesis at the caprolactam moiety, guided by previous studies ${ }^{[8,21]}$ that reported a significant increase in potency by adding lipophilic residues on the heterocycle. The introduction of $N$-benzylic residues with different ring substitutions to yield 7a-e (Figure 1, Scheme S1) in fact led to $\mathrm{IC}_{50}$ values that were 50-230-fold lower than that of 3 (Table S5). However, the utility of 7a-e as drugs was limited by their high lability when incubated with liver microsomes, as exemplified by 7a (Table 1). Not surprisingly, the high metabolic lability in vitro (e.g. $88-99 \%$ for 7a) translated to poor pharmacokinetic properties in vivo: 7a showed an extensive clearance (7.1 $\left.\mathrm{Lh}^{-1} \mathrm{~kg}^{-1}\right)$, a limited volume of distribution $\left(0.9 \mathrm{~L} \mathrm{~kg}^{-1}\right)$, and a short terminal half-life of $0.1 \mathrm{~h}$ (Table 1).

We intended to enhance the stability of substituted bengamides through a migration of the aromatic residue from its benzylic position to the caprolactam ring, thereby resulting in benzo-fused caprolactams. A semisynthetic access to such analogues is conceivable by combining a microbially produced polyketide side chain with synthetic caprolactams. However, to gain fast access to the analogues 8a-f (Figure 1, Scheme S2) at this exploratory stage, an efficient total synthesis route $^{[7]}$ was followed (Scheme S2). From this small series, $8 \mathbf{a}$ and $\mathbf{8 d}$ were selected because of their superior potency and were further profiled against a spectrum of 14 cancer cell lines and nonproliferating peripheral blood lymphocytes (PBLs; Table 2 and Table S6) in comparison to 2. All three compounds inhibited cell proliferation in the nanomolar range, with $\mathbf{2}$ being most potent. Although 8a and 8d were particularly active $\left(\mathrm{IC}_{50}\right.$ values $\left.\leq 59 \mathrm{~nm}\right)$ towards HCT116, B16-F10, H460, and A549 cell lines, the reduced potency against MDA-A1 and HCT15 indicated that 2, 8a, and to a lesser extent $\mathbf{8 d}$ were recognized by the P-glycoprotein (PgP) efflux pump. The fact that the viability of quiescent PBLs was almost unaffected by bengamides illustrates their potential to selectively target dividing tumor cells.

Analogues $8 \mathbf{a}$ and $\mathbf{8 d}$ were stable in plasma and had low metabolic labilities of $5 \% / 0 \% / 0 \%$ and $0 \% / 5 \% / 15 \%$, respec-

Table 1: Metabolic labilities in liver microsomes and pharmacokinetic parameters in C57BL mice of $7 \mathrm{a}, 8 \mathrm{a}$, and $8 \mathrm{~d}$.

\begin{tabular}{|c|c|c|c|c|c|c|c|}
\hline \multirow[t]{3}{*}{ Cmpd } & \multicolumn{4}{|c|}{ Pharmacokinetics in vivo ${ }^{[a]}$} & \multicolumn{3}{|c|}{ Microsomal lability in vitro } \\
\hline & \multirow{2}{*}{$\begin{array}{c}\mathrm{AUC} \\
{\left[\mathrm{h} n g \mathrm{~mL}^{-1}\right]}\end{array}$} & \multirow{2}{*}{$\begin{array}{c}\mathrm{Cl} \\
{\left[\mathrm{Lh}^{-1} \mathrm{~kg}^{-1}\right]}\end{array}$} & \multirow{2}{*}{$\begin{array}{c}V_{\mathrm{dss}} \\
{\left[\mathrm{Lkg}^{-1}\right]}\end{array}$} & \multirow{2}{*}{$\begin{array}{l}t_{1 / 2} \\
{[\mathrm{~h}]}\end{array}$} & \multicolumn{3}{|c|}{ lability in \% } \\
\hline & & & & & mouse & rat & human \\
\hline $7 \mathrm{a}^{[\mathrm{b}]}$ & 140 & 7.1 & 0.9 & 0.1 & 88 & 99 & 94 \\
\hline $8 a^{[c]}$ & 26254 & 1.1 & 1.6 & 3.4 & 5 & 5 & 0 \\
\hline $8 d^{[c]}$ & 25600 & 1.2 & 0.5 & 3.5 & 0 & 5 & 15 \\
\hline
\end{tabular}

[a] $\mathrm{AUC}=$ area under the concentration/time curve, $\mathrm{Cl}=$ clearance,

$V_{\mathrm{dss}}=$ distribution volume, $t_{1 / 2}=$ terminal half-life. [b] $1 \mathrm{mg} \mathrm{kg}^{-1}$ single dose, intravenous administration. [c] $30 \mathrm{mg} \mathrm{kg}^{-1}$ single dose, intravenous administration. 
Table 2: Cellular antiproliferative activities of bengamide analogues $8 \mathrm{a}$ $8 \mathrm{~d}$, and $\mathbf{2}$ (given as $I C_{50}$ values in $n \mathrm{M}$ ). ${ }^{[\mathrm{b}]}$

\begin{tabular}{lcccccc}
\hline & \multicolumn{2}{c}{$8 \mathrm{8a}$} & \multicolumn{2}{c}{$8 \mathrm{~d}$} & \multicolumn{2}{c}{2} \\
cell line & $\mathrm{IC}_{50}$ & s.d. $^{[\mathrm{a}]}$ & $\mathrm{IC}_{50}$ & s.d. & $\mathrm{IC}_{50}$ & s.d. \\
\hline A549 & 9 & 7 & 39 & 23 & 13 & 10 \\
B16-F10 & 33 & 22 & 47 & 24 & 29 & 8 \\
H460 & 59 & 24 & 42 & 26 & 9 & 6 \\
HCT116 & 44 & 29 & 51 & 24 & 23 & 27 \\
HCT15 & 550 & 510 & 45 & 9 & 1300 & 1500 \\
MDA-A1 & 4800 & 2200 & 1100 & 500 & $>10000$ & \\
PBL & $>5900$ & 4400 & & & $>7900$ & 3800
\end{tabular}

[a] s.d. = standard deviation; the incubation time was $96 \mathrm{~h}$ in all cases.

tively, when subjected to mouse, rat, or human liver microsomes (Table 1). This translated to improved pharmacokinetic properties in vivo: 8a showed moderate clearance $\left(1.1 \mathrm{~L} \mathrm{~h}^{-1} \mathrm{~kg}^{-1}\right)$, a moderate volume of distribution $\left(1.6 \mathrm{~L} \mathrm{~kg}^{-1}\right)$, and a terminal half-life of $3.4 \mathrm{~h}$. For comparison, plasma levels of $\mathbf{2}$ - the "gold standard" among bengamidescould not be detected after the first time point (ca. $6 \mathrm{~min}$ ), as $\mathbf{2}$ is a rapidly cleaved ester prodrug.

In an enzymatic assay using manganese as a cofactor, the molecular target MetAP2 was inhibited by $\mathbf{8 a}$ with an $\mathrm{IC}_{50}$ value of $42 \mathrm{~nm}$. The equilibrated solubility of $\mathbf{8 a}$ at pH 7.4 was $370 \mu \mathrm{M}$. At a concentration of $10 \mu \mathrm{M}$, inhibition of $\mathrm{hErg}$, Cyp isoforms, or an AMES signal were not observed.

We finally evaluated the efficacy of $\mathbf{8 a}$ in female C57BL/6 mice bearing early stage B16 melanoma. The highest nontoxic dose $\left(60 \mathrm{mg} \mathrm{kg}^{-1}\right.$ per injection, total dose $\left.480 \mathrm{mg} \mathrm{kg}^{-1}\right)$ had an antitumor activity with a tumor/control (T/C) value of $31 \%$ (Table S7, Figure S12), thereby providing a proof-of-concept for the antitumor activity of caprolactam-modified bengamides in vivo. However, the activity was moderate compared to the reference drug docetaxel (T/C of $1 \%$ ), and the therapeutic window between toxicity and antitumor efficacy was limited.

Most studies on pharmaceutical applications of bengamides explored their anticancer and antimycobacterial potential. As recent preclinical and clinical studies with a covalently binding fumagillin analogue linked MetAP2 inhibition to increased fat catabolism and a strong reduction of body weight, ${ }^{[2]}$ an intriguing future direction may be the exploration of MetAP2-selective bengamides ${ }^{[23]}$ as noncovalently binding anti-obesity agents.

In summary, we conducted detailed studies on the biosynthetic gene cluster encoding for bengamide biosynthesis, thereby enabling its heterologous expression in a more suitable host and also disclosing the bacterial self-resistance mechanism of the producer. The drug properties of the compounds were optimized by semi- and total synthesis, which yielded bengamides with an improved pharmacokinetic profile and efficacy in an antitumor model without the need for a prodrug (as in 2). With the genetic and chemical tools in hand, genetic engineering and synthetic approaches for the cost-effective production of optimized bengamides could be merged, for example, through coupling a biotechnologically generated polyketide chain with a synthetically modified caprolactam.

\section{Acknowledgements}

We thank Dr. Heinrich Decker, Dr. Claus Lattemann, and Dr. Ulrike Jannsen for ensuring the resupply of bengamides. We also thank Valerie Vin for conducting the MetAP inhibition assay and Benoît Pasquier for his evaluation on PBL cells. We thank Bryan Julien for providing plasmid pKOS375-151.1. This work was generously supported by a grant from the German Federal Ministry of Education and Research to Saarland University and Sanofi Aventis (FKZ: 0315198).

Keywords: bengamides - biosynthesis - drug discovery natural products $\cdot$ resistance

How to cite: Angew. Chem. Int. Ed. 2015, 54, 15560-15564 Angew. Chem. 2015, 127, 15781-15785

[1] a) D. J. Newman, G. M. Cragg, J. Nat. Prod. 2012, 75, 311-335; b) A. Bauer, M. Brönstrup, Nat. Prod. Rep. 2014, 31, 35-60; c) M. S. Butler, A. A. Robertson, M. A. Cooper, Nat. Prod. Rep. 2014, 31, 1612-1661.

[2] a) J. W. Blunt, B. R. Copp, R. A. Keyzers, M. H. Munro, M. R. Prinsep, Nat. Prod. Rep. 2015, 32, 116-211; b) T. F. Molinski, D. S. Dalisay, S. L. Lievens, J. P. Saludes, Nat. Rev. Drug Discovery 2009, 8, 69-85.

[3] K. L. Jackson, J. A. Henderson, A. J. Phillips, Chem. Rev. 2009, 109, 3044-3079.

[4] C. Cuevas, A. Francesch, Nat. Prod. Rep. 2009, 26, 322-337.

[5] a) T. L. Simmons, R. C. Coates, B. R. Clark, N. Engene, D. Gonzalez, E. Esquenazi, P. C. Dorrestein, W. H. Gerwick, Proc. Natl. Acad. Sci. USA 2008, 105, 4587 -4594; b) T. Hoffmann, S. Müller, S. Nadmid, R. Garcia, R. Müller, J. Am. Chem. Soc. 2013, 135, 16904-16911.

[6] a) E. Quiñoà, M. Adamczeski, P. Crews, G. J. Bakus, J. Org. Chem. 1986, 51, 4494-4497; b) M. Adamczeski, E. Quiñoà, P. Crews, J. Org. Chem. 1990, 55, 240-242; c) C. García-Ruiz, F. Sarabia, Mar. Drugs 2014, 12, 1580-1622.

[7] D. D. Xu, O. Repič, T. J. Blacklock, L. Waykole, J. V. Calienni, L. Ciszewski, G. T. Lee, W. Liu, J. Szewczyk, K. Vargas, K. Prasad, Org. Process Res. Dev. 2003, 7, 856-865.

[8] a) F. R. Kinder, Jr., et al., J. Med. Chem. 2001, 44, 3692-3699; b) Z. Thale, F. R. Kinder, K. W. Bair, J. Bontempo, A. M. Czuchta, R. W. Versace, P. E. Phillips, M. L. Sanders, S. Wattanasin, P. Crews, J. Org. Chem. 2001, 66, 1733-1741.

[9] H. Dumez, H. Gall, R. Capdeville, C. Dutreix, A. T. Van Oosterom, G. Giaccone, Anticancer Drugs 2007, 18, 219-225.

[10] H. Towbin, et al., J. Biol. Chem. 2003, 278, 52964-52971.

[11] J. L. Mauriz, J. Martin-Renedo, A. Garcia-Palomo, M. J. Tunon, J. Gonzalez-Gallego, Curr. Drug Targets 2010, 11, 1439-1457.

[12] J. P. Lu, X. H. Yuan, H. Yuan, W. L. Wang, B. Wan, S. G. Franzblau, Q. Z. Ye, ChemMedChem 2011, 6, 1041-1048.

[13] a) F. Sarabia, F. Martin-Galvez, C. Garcia-Ruiz, A. SanchezRuiz, C. Vivar-Garcia, J. Org. Chem. 2013, 78, 5239-5253; b) P. K. Metri, R. Schiess, K. R. Prasad, Chem. Asian J. 2013, 8 , 488-493; c) W. Zhang, Q. Liang, H. Li, X. Meng, Z. Li, Tetrahedron 2013, 69, 664-672.

[14] H. Hoffmann, S. Haag-Richter, M. Kurz, H. Tietgen, 2005, WO 2005/044803.

[15] a) J. Zhang, Y. Benedetti, A. Commercon, WO2007/135295; b) J. Zhang, N. Bhatnagar, WO2006/056695.

[16] T. A. Johnson, J. Sohn, Y. M. Vaske, K. N. White, T. L. Cohen, H. C. Vervoort, K. Tenney, F. A. Valeriote, L. F. Bjeldanes, P. Crews, Bioorg. Med. Chem. 2012, 20, 4348-4355.

[17] S. C. Wenzel, R. Müller, Curr. Opin. Drug Discovery Dev. 2009, $12,220-230$. 


\section{Angewandte}

Communications

[18] C. Hertweck, Angew. Chem. Int. Ed. 2009, 48, 4688-4716; Angew. Chem. 2009, 121, 4782-4811.

[19] M. A. Marahiel, J. Pept. Sci. 2009, 15, 799-807.

[20] Y. A. Chan, A. M. Podevels, B. M. Kevany, M. G. Thomas, Nat. Prod. Rep. 2009, 26, 90.

[21] G. Liu, Y. M. Ma, W. Y. Tai, C. M. Xie, Y. L. Li, J. Li, F. J. Nan, ChemMedChem 2008, 3, 74-78.

[22] a) D. D. Kim, J. Krishnarajah, S. Lillioja, F. de Looze, J. Marjason, J. Proietto, S. Shakib, B. G. Stuckey, J. E. Vath, T. E.
Hughes, Diabetes Obes. Metab. 2015, 17, 566-572; b) A. A. Joharapurkar, N. A. Dhanesha, M. R. Jain, Diabetes Metab. Syndr. Obes. 2014, 7, 73-84.

[23] S. Kim, K. LaMontagne, M. Sabio, S. Sharma, R. W. Versace, N. Yusuff, P. E. Phillips, Cancer Res. 2004, 64, 2984-2987.

Received: September 4, 2015

Published online: October 30, 2015 